\title{
افنساحـية العـدد
}

\section{نحو بناء خارطة طريق تخول رقمي المنظمات

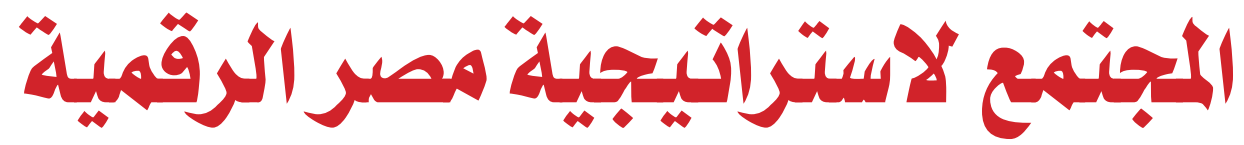

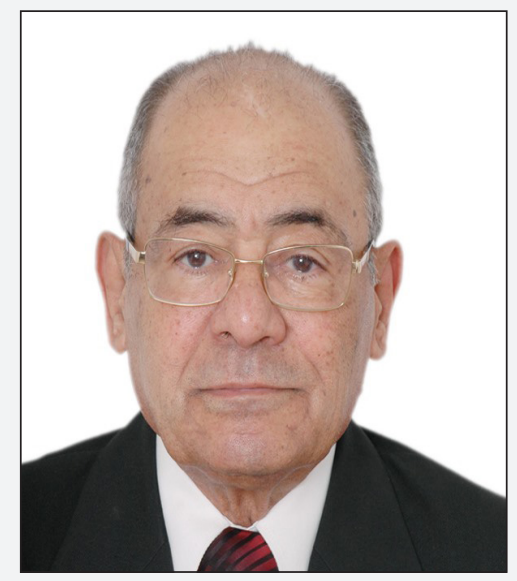

أ.د. محمد محمد الهادي رئيس مجلس إدارة المجلة
في إطار استراتيجية مصر الرقمية الحديثة التي تهف نحو خلق مجتمع مصري متكامل يجب التأكد من أهمية تخطيط ويناء تحول الهية منظمات المجتمع رقميا بطريقة جيدة تعني لبقاء الأعمال المختلفة. وعلي هذا الأساس تصبح خارطة طريق تحول رقيمي معدة جياء طريقا لنجاح نموذج أعمال رقمي لمنظمات المجتمع المصري. وبذلك ينصح بتخطيط سليم للمنظمات المختلفة العديدة بغية إطلاق

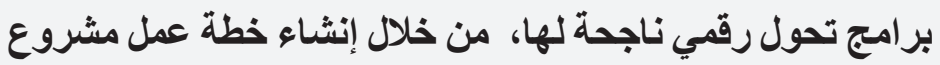
مفصلة تحدد معالم خارطة طريق واضحة لهذه البرامج. و وتعني

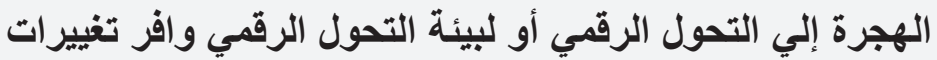
جوهرية مرتبطة بنموذج أعمال المنظمة أو الهيئة أو الثركة الثينة

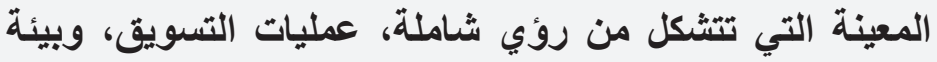
تحتية لتكنولوجيا المعلومات، والعمليات المختلفة.
المنظمة أو الهيئة أو الثركة المعينة التي تتشكل من رؤي شاملة، عمليات التسويق، وبيئة تحتية المناتية لتكنولوجيا المعلومات، و العمليات المختلفة. وفي الواقع، التحول لنموج أعمال رقمي يعتبر

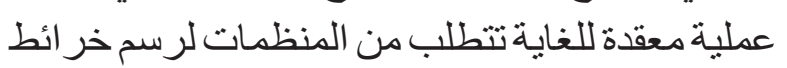

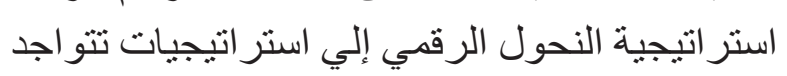

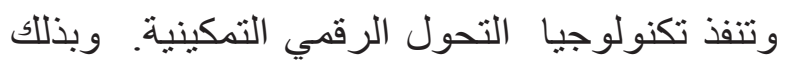

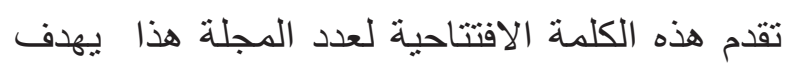

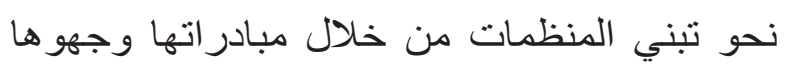

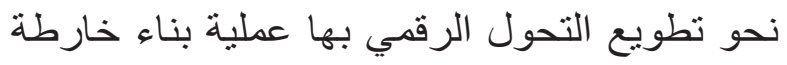
طريق ناجحة وملهمة لكل الإجر اءات المتطلبة. فما هو التحول الرقمي هذا؟ يمكن التفكير

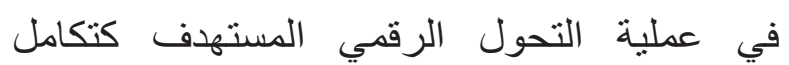

في إطار استر اتيجية مصر الرقمية الحديثة التي تهدف نحو خلق مجتمع مصري متكامل يجب التأكد

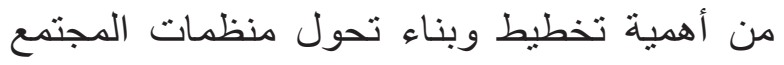
رقميا بطريقة جيدة تعني لبقاء الأعمال المختلفة.

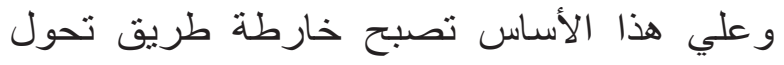

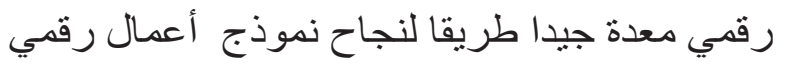
لمنظمات المجتمع المصري. وبذلك ينصح بتخطبط سليم للمنظمات المختلفة العديدة بغية إطلاق بر امج تحول رقمي ناجحة لها،

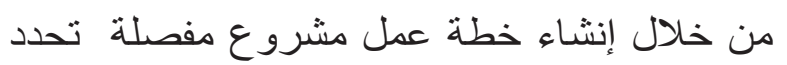

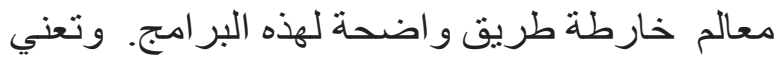
الهجرة إلي التحول الرقمي أو لبيئة التحول الترالت الرقمي وافر تغييرات جوهرية مرتبطة بنموذج أعمال 
و إعادة تصميمه مع إعادة تقديمه أيضـا. وبشكل واضح، فإنه مع اكتساب المزيد من المنظمات الخبرة و إدر الك فو ائد التحول الرقمي فإنها تصبح في وضع يسهم بتبنيهم لفرص سوق أسرع و أكثر نجاحا من الثركات الأخري غير المهتمة لتهم وتتجنب التكنولوجيات الجديدة. وبذلك سوف يحتاج فريق قيادة المنظمة أو الثركة المثكل من أصحاب

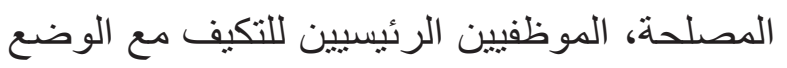
الطبيعي الجديد للأعمال.

من هذا السياق المحدد لفوائد ومزايا التحول

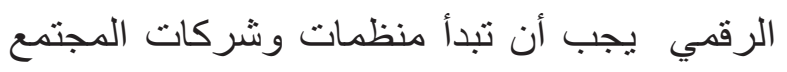

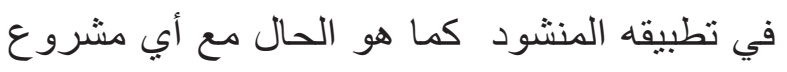
مؤسسي كبير وخاصة تللك المشرو عات المؤثرة علي

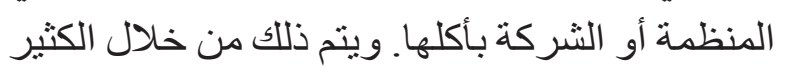

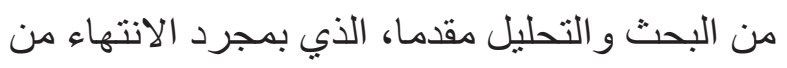
هذا البحث يجب العمل علي إثراك الادارة العليا

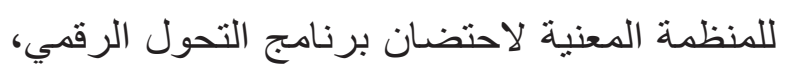

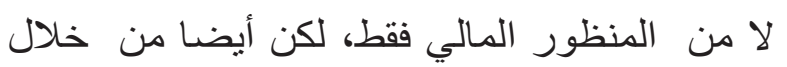
الرؤي الثقافية و التشتيلية.

يلي كل ذلك وضع رؤية و استر اتيجية شاملة للهجرة نحو تبني برنامج التحول الرقمي جنبا إلي ولي ولئي جنب مع خطة مشروع مفصلة تشكل معالم خارطة

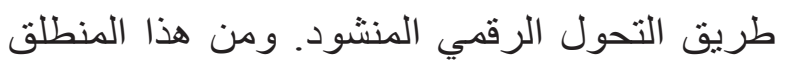

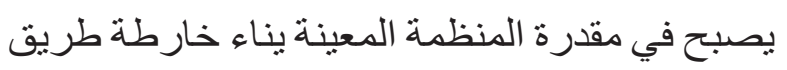
التحول الرقمي التي تتضمن ستة خطو ات رئيسية. وبذلك تستفيد برامج التحول الرقمي الناجحة بشكل فعال من التكنولوجيا والقوي العاملة لتحسين

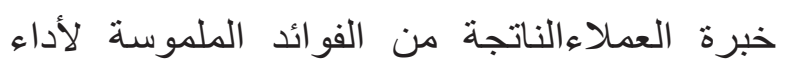

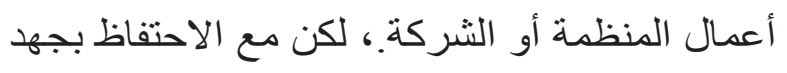

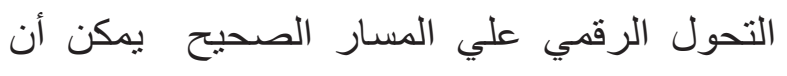
،يكون صعبا لحد ما.

و علي هذا الأساس يقدم هذا الجزء من هذه الورقة
التكنولوجيات المتخصة مثل الذكاء الاصطناعي في كل مجالات الأعمال حيث أن كثير ا إن لم تكن الأن كل الأعمال ستحتاج إلي تغيير جوهري هري وتعتمد علي التكنولوجيات المنشأة الجديدة. كما أن ثقافة لافئ المنظمة أو الثركة من المحتمل أن تتغير أيضا. ويمكن أن يؤدي هذا في تعديل، أو حتي في إلغاء إجر اءات منشأة، وتجربة وندي واعتماد إجر اءات جديدة مع الاستعداد لكل من النجاحات و الفثنل المتوقعة. باختصار ، لكي تحقق الأعمال النجاح مع التحول الرقمي الذي ترتبط كثير من عناصره معا سوف يحتاج إلي التمكن من خطوات جديدة في أنتنشأ وتطبق بعناية. من هذا المنطلق يمكننا التساؤل لماذ يكون التحول

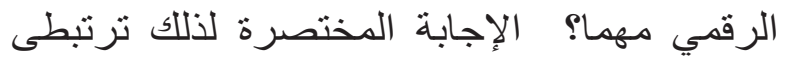
بأهمية بقاء منظمات المجتمع في تأدية وتحسين

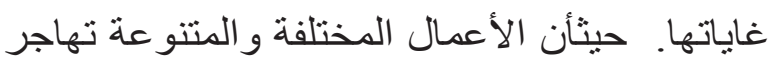
إلي تكنولوجيات وموارد التحول الرقمي من خلان الان قدر اتهم علي زيادة مرلكزهم هو التنافسية، تعزيز سمعاتهم، تعزيز خبرات عملائهم، تحسين الكفاءة

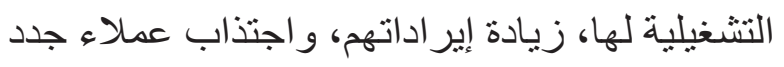

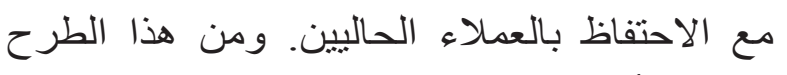

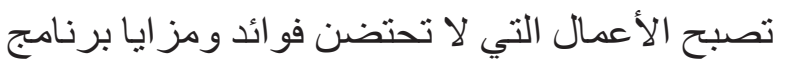
التحول الرقمي مطالبة بضرورة القيام بذلك علي مسئولياتهم الخاصة. وفي نفس الوقت الحالي يمكن ملاحظة أن إنتشار جائحة كوفيدو9 1 تمثل ايضا عاملا جو هريا آخر يحتم تنفيذ المنظمات و الثركات براته امج التحول الرقمية لاتاحة الفرصة من أجل أن يعمل العديد من

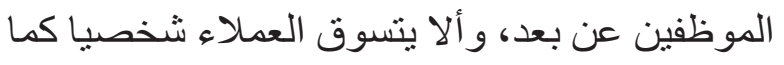

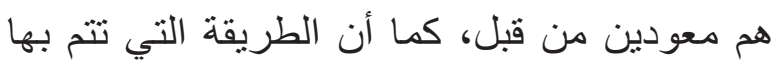

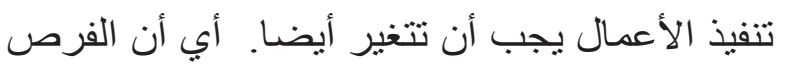

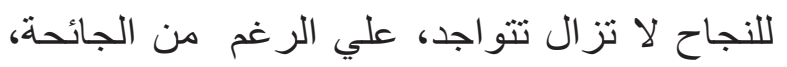

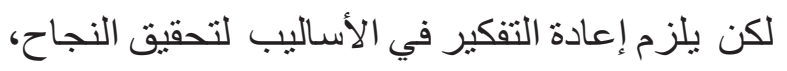


محتاج له وكيفية الانتهاء منها بنجاح يعود بالنفع علي

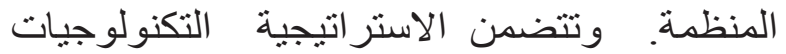
الموظفة، كيفية حدوث الهجرة الموقعة، الأنشطة المتوازية كتدريب الموظفين وتعديلات البنية التحتية للتكنولوجيا المحتاج لها، مع كافة الأنشطة الأخري

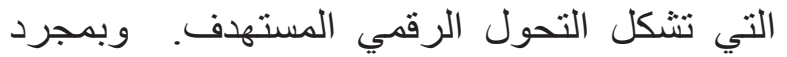
وصف وتحديد كل ذلك، تتمثل الحركة التالية في إيجاز صفات برنامج التحول الرقمي للإدارة العليا و الموظفين، وتعديل الاستر اتيجية ومساندة الأنشطة

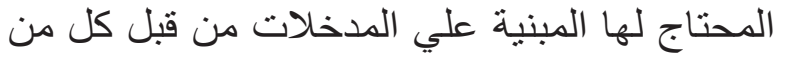
الإدارة العليا وباقي موظفي المنظمة الرئيسيين. ثُالثا: تعريف خيارات التكنولوجيا، تأمين موافقة الإدارة العليا والتمويل: كجزء من تطوير

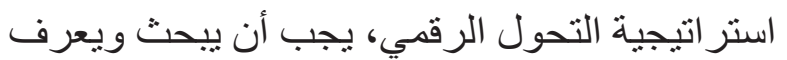
فريق المشروع كل من النظم والتكنولوجيات،

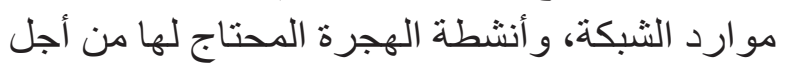

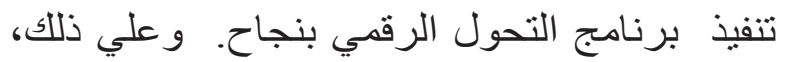
يجب معرفة كل التكاليف المرتبط بالهجرة نحون التحول الرقمي المنشود التي تتضمن التكنولوجيات الجديدة، تحديث النظم القائمة، التطبيقات الجديدة و النظم المتخصصة كما في حالة استخدام الذكاء الاصطناعي، الخبرة الداخلية و الخارجية، تعديلات التسهيلات الطبيعية لاستيعاب المكونات الجديدة وغير ذللك من معلومات يحتاج إليها. وبمجرد جمع هذه المعلومات يجب أن تراجع ويو افق عليها بو اسطة الخبرة المناسبة، ومو افقة المدر اء الملائمين

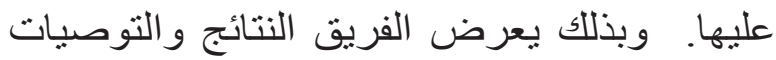
المتوصل لها علي الإدارة العليا للمنظمة بغية لئنية المو افقة عليها.

رابعا: الموافقة علي خطة مشروع التحول الرقمي وإطلاقه: في هذه الخطوة، يتم إحضار كل

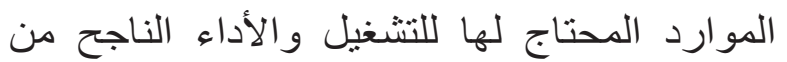

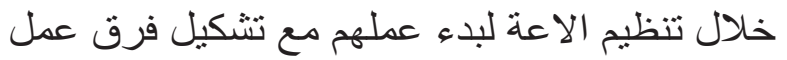

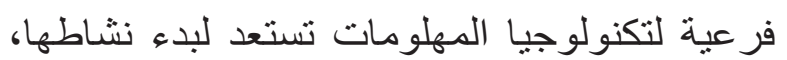

البحثية ستة خطوات أساسية تتشكل منها خارطة طريق التحول الرقمي التي يجب أن تعيها وتحتذي بها منظمات المجتمع المصري في إطار استر اتيجية مصر الرقمية وتساعدها تأكيد برامج تحول فئر رقمي

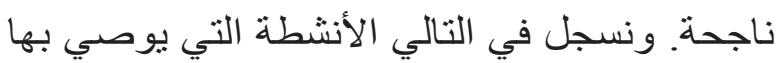
و الأطر الزمنية النسبية، ومبادر ات الحدود الرئيسية التئية

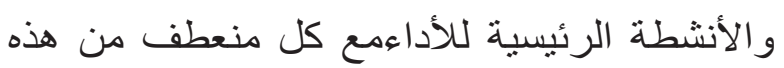
الخطوات الستة بحيث تبني كل خطوة علي الأفعال المكتملة في الخطوة السابقة. وفيما يلي استعر اض الت التعال موجز لهذه الخطوات الستة المشكلة لخارطة طريق التحول الرقمي الناجح التي يجب أن تقوم يها مؤسسات المجتمع المصري لتحقيق استر اتيجية

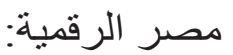

أولا: أداء البحث والعناية الواجبة: تشكيل فريق عمل مشروع التحول الرقمي يتم في هذا التها

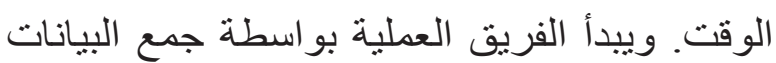

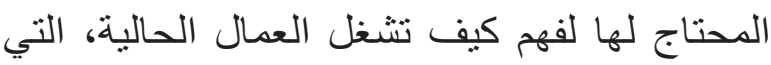
تتضمن الموارد المستخدمة بواسطة الأعمال مثل

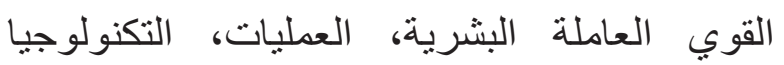
و التسهيلات اللازمة. و علي الفريق مر اجعة خطط المنظمة الاستر اتيجية الحالية، الخطط الاستر اتيجية

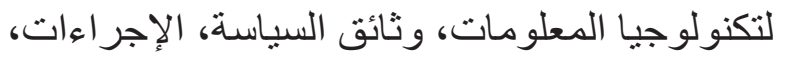

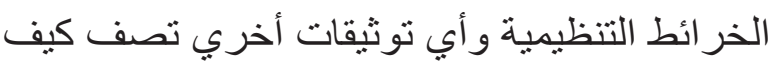
تشغل الأعمال. يلي ذللك، يجب علي التئ الفريق مقابلة

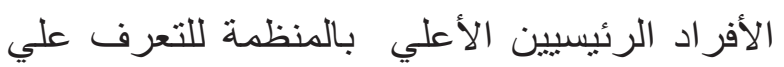

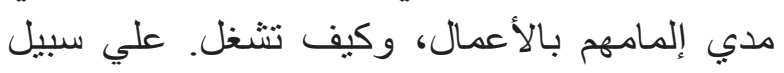
المثال، تتشكل افراد المنظمة الرئيسيين من كبار

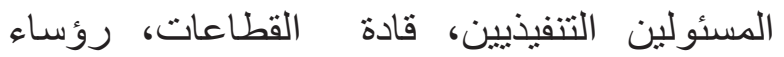
الإدارات والأقسام، مع خبراء الدجالات المختاءتلفة.

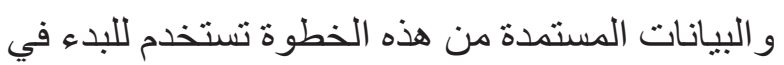
أنشطة الخطوة التالية.

ثانيا: إعداد استراتيجية التحول الرقمي وإيجازها للإدارة العليا والموظفين بالمنظمة: استراتيجية التحول الرقمي يجب أن تصف ابعادو وغايات ما هو 
و عمل التعديلات كلما احتاج لها.

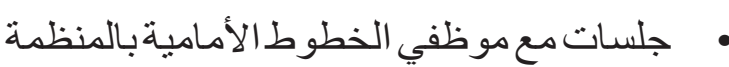

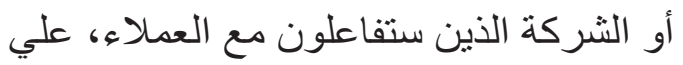
سبيل المثال خلال تجريب البرامج لتأكيد أنها مريحة وسهلة مع التكنولوجيا الجديدة ويمكنها تقديم تحويل رقمي متقدم ومعزز . قياسات لحماية النظم و التكنولوجيات الجديدة، علي سبيل المثال، خطط التعافي من الكو ارث

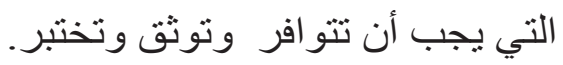
إجراءات نسخ البيانات احتياطيا والحصول

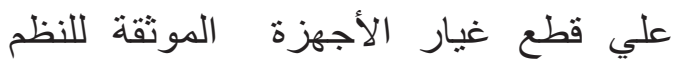
و التطبيقات الجديدة.

• البرامج التي تؤكد استخدام الموظفين التكنولوجيا الجديدة من أجهزة مناسبة وأماكن الترائ

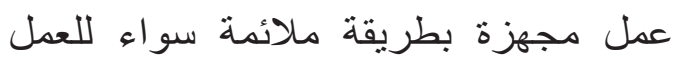
داخل الموقع أو عن بعد. • خطة للعيش تتضمن مو عد مباشر مع تو اريخ بديلة مع كيفية تنفيذ عملية الانتقال المبانشر. • تسويق موقع الويب والأنشطة الترويجية الأخري بغية الحصول علي خارج الكلمة

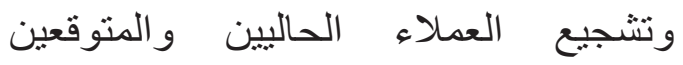
لاستخدام النظام أو التطبيق الجديد.

سادسا: بدء خيارات الأعمال المحدثة والأداء:

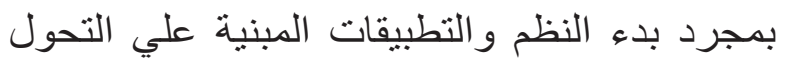

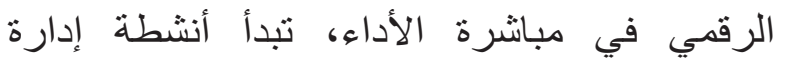

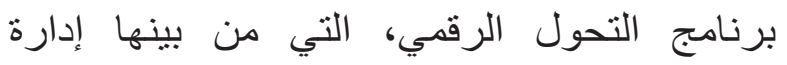

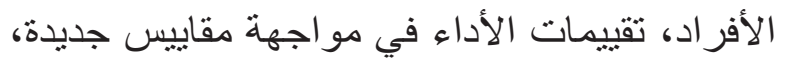

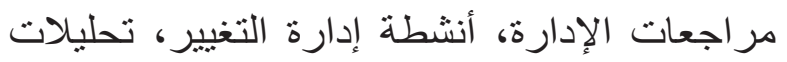

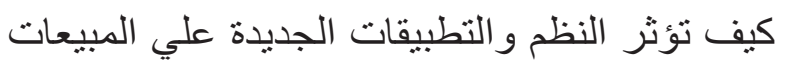

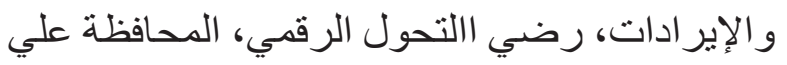

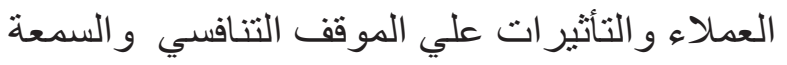
للمنظمة أو الثركة.
وإعداد الموظفين للبدء في التحول من عملياتهم

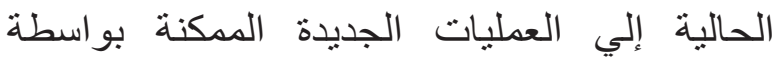
برنامج التحول الرقمي. وتعتبر هذه الخطوة من التحان

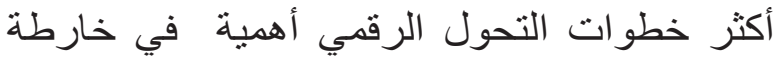
طريق التحول الرقمي حيث تجمع كل المشتركين معاوتؤدي لتحرك المشروع إلي الأمام.

خامسا: تنفيذ أنشطة الهجرة والتحول المستهدفة: خلال عملية تنفيذ برنامج التحول الرقمي، توجد أنشطة متعددة يجب ان ان يتم تنفيذها. وتتضمن هذه النشطة العديدة علي التالي:

• تركيب واختبار الأجهزة و البرمجيات الجديدة

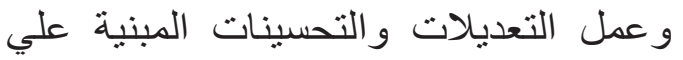

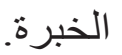

تجريب التكنولوجيات الجديدة لتعريف الموظفين بالنظم والتطبيقات وكيفية استخدامها بعناية. وسوف يساعد التجريب في تطوير السياسات، الإجر اءات، وقياسات الأداء لاستخدام سياسات الموارد البشرية الإسية

$$
\text { عندما يحتاج لها. }
$$

برامج التدريب لكل الأفراد بالمنظمة عن النظم والتطبيقات الجديدة المستحدثة وخاصة للموظفين الذين يستخدمونها. مر اجعات منتظمة لنفقات النظم و التطبيقات مقابل الميزانيات مع التعديلات المتطلبة عند الحاجة

إختبار ات التكنولوجيا المتقدمة التجريبية، علي سبيل المثال الذكاء الاصطناعي وتعلم الآلة لرؤية كيف تكون قدراتها أحسن استخداما لتحليل البيانات المنشأة بواسطة لكأنة النظم و التطبيقات الجديدة. • إختبارات المخرجات من النظم و التطبيقات الجديدة لتأكيد أن النتائج منو افقة مع المنظمة ولنيات 